\section{Local Persistence of Novel MRSA Lineage after Hospital Ward Outbreak, Cambridge, UK, 2011-2013}

\author{
Michelle S. Toleman, Sandra Reuter, \\ Francesc Coll, Ewan M. Harrison, \\ Sharon J. Peacock
}

Author affiliations: University of Cambridge Department of Medicine, Addenbrooke's Hospital, Cambridge, UK

DOI: http://dx.doi.org/10.3201/eid2209.151100

To the Editor: Previously, we reported the use of whole-genome sequencing to investigate a putative methicillin-resistant Staphylococcus aureus (MRSA) outbreak in 2011 in the special care baby unit (SCBU) at the Cambridge University Hospitals National Health Service Foundation Trust (CUH) in the United Kingdom (1). The report identified 26 related cases of infection with or asymptomatic carriage of MRSA and showed that transmission occurred within the SCBU, between mothers on a postnatal ward, and in the community; the outbreak apparently resolved at the end of 2011. The outbreak strain, sequence type (ST) 2371, was of a novel multilocus ST related to the dominant hospital-associated lineage in the UK (ST22, EMRSA-15), but unlike most ST22 strains, this strain was Panton-Valentine leucocidin-positive (2). Since then, ST2371 has been identified as a prevalent communityassociated MRSA clone in Southern India, and sporadic isolates have also been detected by whole-genome sequencing of MRSA in Denmark (3-5).

During April 2012-April 2013, we implemented genomic surveillance of MRSA isolated at the diagnostic microbiology laboratory at the CUH (F. Coll, unpub. data). From this, we noted that 10 isolates cultured from samples submitted from general practice $(\mathrm{n}=7)$ and hospital wards $(\mathrm{n}=3)$ during June 2012-February 2013 were classified as ST2371. Phylogenetic comparison between these 10 isolates and the 45 isolates from the original outbreak demonstrated that these strains were highly related (staphylococcal cassette chromosome mec IVc, Panton-Valentine leucocidin-positive, staphylococcal protein A $[s p a]$ type 852) (Figure)

We undertook an epidemiologic investigation to determine whether links could be identified between these new cases and the original outbreak. The 10 isolates were cultured from 5 patients (case-patients A-E), all of whom had a direct or indirect link to the 2011 outbreak. Case-patients from the 2011 outbreak are identified by the alphanumeric code assigned during that outbreak investigation (e.g., P22) (1).

Case-patients A and B were also case-patients in the original SCBU outbreak (P22 on the postnatal ward and P14 in the SCBU, respectively). Case-patient $\mathrm{C}$ was born at the CUH and was not screened for MRSA, but both parents were case-patients in the SCBU outbreak (P20 and P26). Case-patient $\mathrm{D}$ was born at the $\mathrm{CUH}$ and discharged when 5 days old, which was 2 days before the birth of the presumed index case-patient of the original SCBU outbreak. The sample for the first isolate from case-patient D was collected almost 2 years later; acquisition could have occurred at the

Figure. Midpoint-rooted phylogenetic tree based on single-nucleotide polymorphisms (SNPs) in the core genome of methicillinresistant Staphylococcus aureus isolates from 2 investigations in the United Kingdom in 2011 and 2012-2013. Isolates were mapped against the EMRSA-15 reference genome. Open circles denote 20 individual colonies from a nasal swab culture taken from a healthcare worker during an outbreak in a hospital special care baby unit (SCBU) in 2011. Gray shaded circles denote isolates from 25 patients and their family members investigated during the 2011 outbreak. Black circles denote 10 isolates from 5 persons (case-patients $A-E$ ) from whom microbiological samples were taken during the 2012-2013 study. Numbers prefixed by $P$ indicate the original study number used for each case during the 2011 outbreak investigation. Multiple samples from the same patient are identified by an underscore followed by the sample number. Two casepatients (P22/A and P14/B) were included in both outbreaks. Scale bar indicates SNPs.

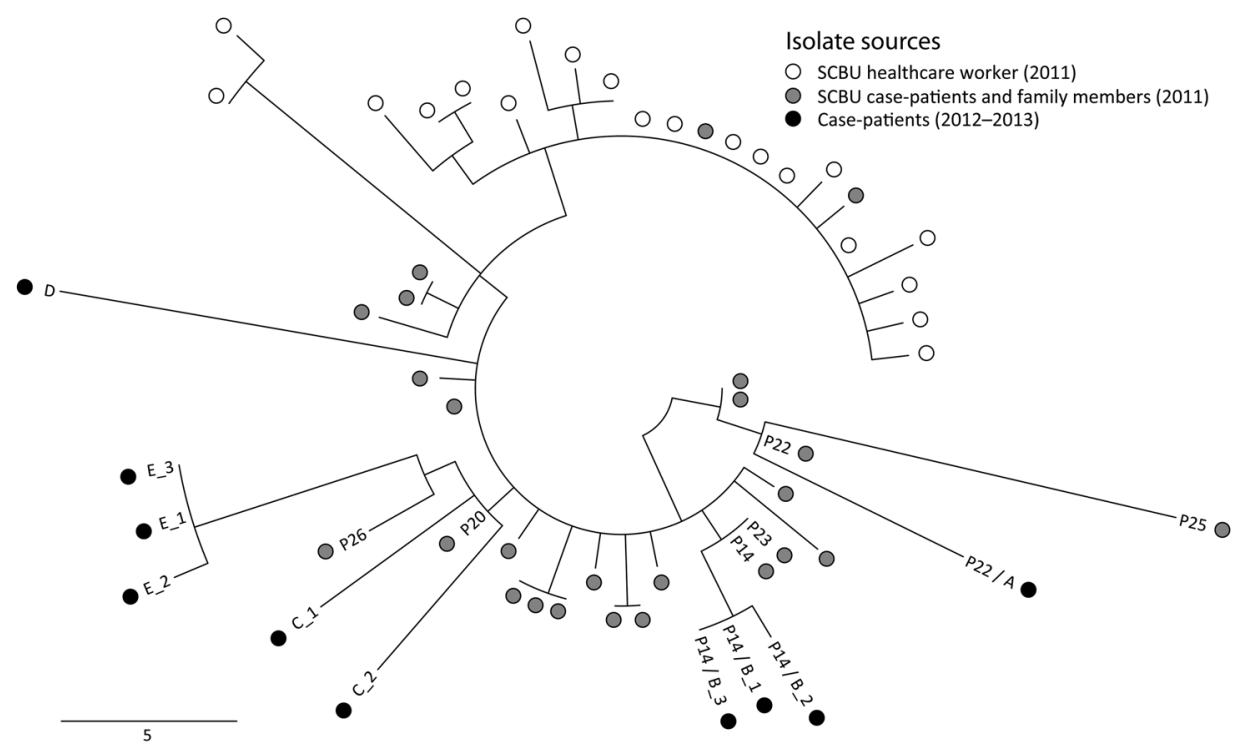


CUH or from subsequent contact with unsuspected carriers in the case-patient's family or the community. On the basis of a matching surname, case-patient $\mathrm{E}$ was determined to be a member of the same family as case-patients P20, P26, and likely C. Soft tissue infection was documented in all 5 casepatients, supporting the original observation that ST2371 is associated with disease. Evidence of familial transmission in the original outbreak is further supported by transmission between case-patients P20, P26, C, and E. Furthermore, 2 case-patients infected during the original outbreak, P22/A and $\mathrm{P} 14 / \mathrm{B}$, continued to experience disease signs and symptoms for $\geq 15$ months after their initial diagnosis.

Our data highlight the role of hospitals as reservoirs of MRSA and subsequent failure to track the entry and spread of MRSA in the community. MRSA decolonization was advised in all cases in the original outbreak, but this process clearly proved ineffective for case-patients A and B. Potential explanations include not implementing or completing the course of decolonization; failed decolonization; or limiting decolonization to only some members of an affected family. Although the outbreak in the hospital ward was resolved, the lack of a systematic surveillance program to monitor the incidence of noninvasive MRSA infections among the case-patients' contacts and the community allowed this novel lineage to continue to cause disease in a group of linked persons. Considering recommendations to move from universal to targeted MRSA screening in hospitals in England (6), more active surveillance of any identified case-patients or carriers of MRSA in the community may be warranted.

This study was supported by grants from the UKCRC Translational Infection Research (TIR) Initiative, and the Medical Research Council (grant no.G1000803) with contributions to the Grant from the Biotechnology and Biological Sciences Research Council, the National Institute for Health Research on behalf of the Department of Health, and the Chief Scientist Office of the Scottish Government Health Directorate (to S.J.P.); by a Healthcare Infection Society Major Research Grant; and by Wellcome Trust grant no. 098051 awarded to the Wellcome Trust Sanger Institute.

M.S.T., F.C., E.M.H., and S.R. undertook epidemiological and bioinformatic analysis of whole-genome sequence data. S.R. prepared the figure. S.J.P. supervised and managed the study. All authors were involved in compiling the report and approved the final version.

\section{References}

1. Harris SR, Cartwright EJP, Török ME, Holdern MT, Brown NM, Ogilvy-Stewart AL, et al. Whole-genome sequencing for analysis of an outbreak of meticillin-resistant Staphylococcus aureus: a descriptive study. Lancet Infect Dis. 2013;13:130-6. http://dx.doi.org/10.1016/S1473-3099(12)70268-2

2. Grundmann H, Aanensen DM, van den Wijngaard CC, Spratt BG, Harmsen D, Friedrich AW, et al. Geographic distribution of
Staphylococcus aureus causing invasive infections in Europe: a molecular-epidemiological analysis. PLoS Med. 2010;7:e1000215. http://dx.doi.org/10.1371/journal.pmed.1000215

3. Rajan V, Schoenfelder SM, Ziebuhr W, Gopal S. Genotyping of community-associated methicillin resistant Staphylococcus aureus (CA-MRSA) in a tertiary care centre in Mysore, South India: ST2371-SCCmec IV emerges as the major clone. Infect Genet Evol. 2015;34:230-5. http://dx.doi.org/10.1016/j.meegid.2015.05.032

4. Bouchiat C, El-Zeenni N, Chakrakodi B, Nagaraj S, Arakere G, Etienne J. Epidemiology of Staphylococcus aureus in Bangalore, India: emergence of the ST217 clone and high rate of resistance to erythromycin and ciprofloxacin in the community. New Microbes New Infect. 2015;7:15-20. http://dx.doi.org/10.1016/j. nmni.2015.05.003

5. Bartels MD, Larner-Svensson H, Meiniche H, et al. Monitoring meticillin resistant Staphylococcus aureus and its spread in Copenhagen, Denmark, 2013, through routine whole genome sequencing. Euro Surveill. 2015;20:pii:21112.

6. Department of Health expert committee on Antimicrobial Resistance and Healthcare Associated Infection. Implementation of modified admission MRSA screening guidance for NHS (2014) [cited 2015 May 20]. https://www.gov.uk/government/uploads/ system/uploads/attachment_data/file/345144/Implementation of modified_admission_MRSA__screening_guidance_for_NHS.pdf

Address for correspondence: Michelle S. Toleman, Department of Medicine, University of Cambridge, Box 157, Addenbrooke's Hospital, Hills Rd, Cambridge CB2 0QQ, UK; email: mst39@cam.ac.uk

\section{Community-Acquired Clostridium difficile Infection, Queensland, Australia}

\section{Luis Furuya-Kanamori, Laith Yakob, Thomas V. Riley, David L. Paterson, Peter Baker, Samantha J. McKenzie, Jenny Robson, Archie C.A. Clements}

Author affiliations: The Australian National University, Canberra, Australian Capital Territory, Australia (L. Furuya-Kanamori, A.C.A. Clements); London School of Hygiene and Tropical Medicine, London, UK (L. Yakob); The University of Western Australia and PathWest Laboratory Medicine, Nedlands, Western Australia, Australia (T.V. Riley); The University of Queensland, Herston, Queensland, Australia (D.L. Paterson, P. Baker) The University of Queensland, St. Lucia, Queensland, Australia (S.L. McKenzie); Sullivan Nicolaides Pathology, Taringa, Queensland, Australia (J. Robson)

DOI: http://dx.doi.org/10.3201/eid2209.151115

To the Editor: In Queensland, Australia, a steady increase in community-acquired (CA) Clostridium difficile infections (CDI) during 2003-2012 could not be explained by patients' demographic characteristics or environmental 